\title{
The legend of MONARCH: Chinese breast cancer experts from participation in to leading global clinical study and boosting treatment area development
}

\author{
Qian Liu \\ China Medical Tribune, Beijing, China \\ Correspondence to: Qian Liu. Chief Editor of Oncology, China Medical Tribune, Beijing, China. Email: liuq@cmt.com.cn.
}

Received: 08 January 2022; Accepted: 22 January 2022.

doi: $10.21037 /$ tbcr-22-6

View this article at: https://dx.doi.org/10.21037/tbcr-22-6

Breast cancer is the malignant neoplasm with the highest morbidity and mortality among women in the world (1). Among the various subtypes of advanced breast cancer, HR+ and HER2 - are the most common one, accounting for about $60-70 \%$, which are the breast cancer types with the largest proportion (2-4). Professor Sun Yan and Song Santai are committed to the concept of individualized neoplasm treatment and advocate the value of endocrine therapy (ET) for HR+ breast cancer. Currently, ET combined with CDK4 \& 6 inhibitors has been proved to be non-inferior to chemotherapy, with a longer duration. It has been unanimously recommended by domestic and foreign authoritative guidelines (5-7), which has changed the treatment set-up of patients with HR+ and HER2advanced breast cancer. In January of this year, we had an interview with professor Zefei Jiang, the leading principal investigator (PI) of MONARCH plus. He will introduce Abemaciclib following the journey of the monarch serial studies.

\section{Multi-line treatment-MONARCH 1 Study}

The multiple-line treatment of refractory patients is the most challenging one. MONARCH 1 is a phase II single arm study to evaluate the safety and efficacy of Abemaciclib monotherapy in 132 female patients with refractory HR+ and HER2- advanced breast cancer who had progressed on or after prior ET and chemotherapy or advanced breast cancer. The study confirmed that the Abemaciclib monotherapy significantly increased the ORR of refractory patients (8).

\section{Endocrine drug resistance-MONARCH 2 Study}

Although ET does bring many survival benefits, endocrine drug resistance remains an inevitable dilemma for the treatment of breast cancer, which stimulates further investigation in the Abemaciclib study. The MONARCH 2 study is a randomized, open-label, globally multi-centered, and phase III clinical study to evaluate the efficacy and the safety of Abemaciclib combined with fulvestrant, which is compared with the safety of placebo combined with fulvestrant in endocrine drug resistant patients with HR+ and HER2- advanced breast cancer. The study enrolled 669 endocrine drug resistant patients with $\mathrm{HR}+$ and HER2- advanced breast cancer, including premenopausal/ perimenopausal or postmenopausal patients, who had not received chemotherapy for advanced breast cancer, and had not received more than $1 \mathrm{ET}$ for advanced breast cancer. The experimental arm received Abemaciclib combined with fulvestrant, while the control arm was given placebo combined with fulvestrant, and the primary goal of the study was PFS (9). The MONARCH 2 study was initiated in July 2014, and the PFS and ORR data were published on 7CO in 2017 (9). The updated data of OS and PFS were published on European Society for Medical Oncology (ESMO) in 2019, followed by the publication of the full text in 2020 on $7 A M A$ Oncology (10). The study confirmed that the combination of Abemaciclib and fulvestrant significantly prolonged the PFS and OS of endocrine drug 
resistant patients with HR+ and HER2 - advanced breast cancer $(9,10)$.

\section{Endocrine sensitivity-MONARCH 3 Study}

Now, let's focus on the initial endocrine sensitive patients. The MONARCH 3 study is a randomized, open-label, globally multi-centered, and phase III clinical study to evaluate the efficacy and safety of Abemaciclib combined with NSAI, which is compared with the efficacy and safety of placebo combined with NSAI in endocrine sensitive patients with HR+ and HER2 - advanced breast cancer. The study enrolled a total of 493 postmenopausal patients with HR+ and HER2 - advanced breast cancer, who had not received prior systemic therapy, such as neoadjuvant therapy or adjuvant ET, but it had a disease-free interval of more than 12 months, and all subjects were endocrine sensitive patients. The experimental arm received Abemaciclib combined with NSAI, while the control arm was treated with placebo combined with NSAI. The primary goal of the study was PFS (11). The MONARCH 3 study was initiated in December 2014. The PFS data were firstly published on $7 C O$ in 2017 (11), and followed by the publication of final PFS data on NPJ Breast Cancer in 2019 (12). The study confirmed that the combination of Abemaciclib and NSAI significantly prolonged the PFS of endocrine sensitive patients with HR+ and HER2advanced breast cancer $(11,12)$.

\section{Chinese characteristics-MONARCH plus Study}

After introducing foreign clinical data, let's focus on the data of Chinese population. The MONARCH plus study, which led by Professor Hu Xichun and Zefei Jiang, and it is a randomized, open-label, globally multi-centered, and phase III clinical study. This study enrolled 463 patients from 45 medical centers in China, Brazil, India, and South Africa, and Chinese patients accounted for more than $80 \%$. The two cohorts was creative design that patients of the study were divided into two cohorts. Patients enrolled in cohort A were similar to those of MONARCH 3, who were predominantly endocrine sensitive patients with a small number of endocrine drug resistant patients enrolled (about $20 \%$ had previously received tamoxifen and or exemestane). The experimental arm was treated with Abemaciclib combined with NSAI, and the control arm placebo combined with NSAI. Patients enrolled in cohort B corresponded to those of MONARCH 2, who were all endocrine drug resistant patients. The experimental arm received Abemaciclib combined with fulvestrant, and the control arm was given placebo combined with fulvestrant. The primary goal of the study was PFS of cohort A. The interim results announced at the ESMO meeting in 2019 verified the efficacy and safety of CDK4 \& 6 inhibitors combined with ET in the Chinese population for the first time (13). The study results, especially the safety data, are more instructive for Chinese patients. Based on this study, Abemaciclib was approved for indications in China on December. 29, 2020, in combination with fulvestrant and AI simultaneously (14). As of today, there are still patients in clinical trials who continue to benefit from Abemaciclib therapy.

\section{Adjuvant therapy—monarchE Study}

After the significant benefit of Abemaciclib in HR+, HER2 - advanced breast cancer had been confirmed, clinical studies of Abemaciclib in early-stage breast cancer were carried out to explore its clinical value in adjuvant therapy. The MonarchE study is a randomized, openlabel, globally multi-centered, and phase III clinical study and it enrolled patients with HR+, HER2- early-stage breast cancer at high risk of recurrence, and the subjects received Abemaciclib combined with standard of care adjuvant ET or standard of care adjuvant ET alone. The interim analysis was carried out in March 2020, with a median follow-up time of 15.5 months, and $26.4 \%$ of patients had finished the treatment specified in the study protocol. In June of the same year, it announced that the study has reached the primary goal. In September, the result of the interim analysis was published in ESMO, followed by the publication of the full text on $7 \mathrm{CO}$. After 3.6 months of interim analysis, the main results analysis was performed in July 2020, where $41 \%$ of patients had completed the treatment in the study protocol. The main results (including 2-year IDFS and DRFS) were presented in December of the same year, and the main results of the Chinese subgroup were presented in American Society of Clinical Oncology (ASCO) in June 2021. The followup analysis was conducted in April 2021, with a median follow-up time of 27.1 months, and $89.6 \%$ of patients had completed the treatment specified in the study protocol. The follow-up results (including 3-year IDFS and DRFS) were updated in a virtual online meeting of ESMO, and the full text was published in the Annals of Oncology. A step-by-step disclosure of results has confirmed the stable 
and sustained IDFS and DRFS benefits of Verzenios ${ }^{\circledR}$ combined with ET in the ITT population, and proved that Abemaciclib is the first and only CDK4 \& 6 inhibitor that significantly reduces the risk of recurrence in patients with high-risk HR+, HER2- early-stage breast cancer. The study enrolled 501 Chinese patients (accounting for $9 \%$ ), which about $70 \%$ were from 16 medical centers in Chinese mainland. Based on this, Abemaciclib combined with ET was approved for indications in China on Dec. 31,2021 . The definition of "high risk" is unique to this study, and precise screening of the patient population will be the study direction of early breast cancer, especially in neoadjuvant therapy.

\section{HER2 Positive-monarchHER Study}

In addition to targeting HR+ and HER2- breast cancer, Abemaciclib is also under exploration in the treatment of HR+ and HER2- breast cancer. The MonarchHER study is a randomized phase II study of Abemaciclib plus trastuzumab, with or without fulvestrant, in female patients with HR+ and HER2+ ABC $(\mathrm{N}=237)$ who had received at least 2 HER2-targeted advanced disease treatment. Moreover, ongoing studies of Abemaciclib are exploring the application of Abemaciclib in hormone-related neoplasms, such as prostate cancer, and we look forward to more data release to help more tumor patients in embracing more exciting moments of their lives!

Through international exchanges and collaboration, the development of globally multi-centered clinical studies, and the continuous enrichment of our own clinical experience, professor Zefei Jiang and his colleagues have further improved the diagnosis and treatment guidelines and helped more patients to solve their clinical problems. Recalling the history of breast cancer treatment in the past few decades, the Chinese experts have made great strides from the socalled slash-and-burn era of surgery, chemotherapy, and radiotherapy to the era of precision treatment featuring ET, targeted therapy and immunotherapy. The treatment of breast cancer will become more precise and refined, truly achieving precise stratification and precise treatment. In the future, professor Jiang believed that they will introduce more scientific technologies, such as identifying what kind of patients are suitable for chemotherapy, ET or targeted therapy based on genetic testing. For example, the artificial intelligence-assisted system, developed by professor Jiang and their colleagues over the years, help clinicians dynamically optimize treatment regimens in real time and monitor adverse reactions of the patients. Cultivating more case managers to maintain real-time communication between experts and patients and implement whole-course real-time intelligent case management based on artificial intelligence is also the future direction of development. We hope that with the advancement of science and technology, the survival period of advanced patients will be further prolonged, the cure rate of early-stage patients will be improved, and more breast cancer patients will achieve survival benefits!

\section{Acknowledgments}

I would like to express my heartfelt gratitude to Dr. Zefei Jiang for accepting this interview.

Funding: None.

\section{Footnote}

Provenance and Peer Review: This article was commissioned by the editorial office, Translational Breast Cancer Research. The article did not undergo external peer review.

Conflicts of Interest: The author has completed the ICMJE uniform disclosure form (available at https://tbcr. amegroups.com/article/view/10.21037/tbcr-22-6/coif). The author has no conflicts of interest to declare.

Ethical Statement: The author is accountable for all aspects of the work in ensuring that questions related to the accuracy or integrity of any part of the work are appropriately investigated and resolved.

Open Access Statement: This is an Open Access article distributed in accordance with the Creative Commons Attribution-NonCommercial-NoDerivs 4.0 International License (CC BY-NC-ND 4.0), which permits the noncommercial replication and distribution of the article with the strict proviso that no changes or edits are made and the original work is properly cited (including links to both the formal publication through the relevant DOI and the license). See: https://creativecommons.org/licenses/by-nc-nd/4.0/.

\section{References}

1. Siegel RL, Miller KD, Fuchs HE, et al. Cancer statistics, 2022. CA Cancer J Clin 2022;72:7-33.

2. Deluche E, Antoine A, Bachelot T, et al. Contemporary 
outcomes of metastatic breast cancer among 22,000 women from the multicentre ESME cohort 2008-2016. Eur J Cancer 2020;129:60-70.

3. Howlader N, Cronin KA, Kurian AW, et al. Differences in Breast Cancer Survival by Molecular Subtypes in the United States. Cancer Epidemiol Biomarkers Prev 2018;27:619-26.

4. Vaz-Luis I, Lin NU, Keating NL, et al. Racial differences in outcomes for patients with metastatic breast cancer by disease subtype. Breast Cancer Res Treat 2015;151:697-707.

5. Guidelines Working Committee of Chinese Society of Clinical Oncology. Chinese Society of Clinical Oncology (CSCO) Guidelines for the Diagnosis and Treatment of Breast Cancer. 2021.

6. Cardoso F, Paluch-Shimon S, Senkus E, et al. 5th ESOESMO international consensus guidelines for advanced breast cancer (ABC 5). Ann Oncol 2020;31:1623-49.

7. NCCN Clinical Practice Guidelines in Oncology. Breast Cancer. Version 1. 2022.

8. Dickler MN, Tolaney SM, Rugo HS, et al. MONARCH 1, A Phase II Study of Abemaciclib, a CDK4 and CDK6 Inhibitor, as a Single Agent, in Patients with Refractory HR+/HER2 - Metastatic Breast Cancer. Clin Cancer Res 2017;23:5218-24.

9. Sledge GW Jr, Toi M, Neven P, et al. MONARCH 2: Abemaciclib in Combination With Fulvestrant in Women

doi: $10.21037 /$ tbcr-22-6

Cite this article as: Liu Q. The legend of MONARCH: Chinese breast cancer experts from participation in to leading global clinical study and boosting treatment area development. Transl Breast Cancer Res 2022;3:1.
With HR+/HER2 - Advanced Breast Cancer Who Had Progressed While Receiving Endocrine Therapy. J Clin Oncol 2017;35:2875-84.

10. Sledge GW Jr, Toi M, Neven P, et al. The Effect of Abemaciclib Plus Fulvestrant on Overall Survival in Hormone Receptor-Positive, ERBB2-Negative Breast Cancer That Progressed on Endocrine TherapyMONARCH 2: A Randomized Clinical Trial. JAMA Oncol 2020;6:116-24.

11. Goetz MP, Toi M, Campone M, et al. MONARCH 3: Abemaciclib As Initial Therapy for Advanced Breast Cancer. J Clin Oncol 2017;35:3638-46.

12. Johnston S, Martin M, Di Leo A, et al. MONARCH 3 final PFS: a randomized study of abemaciclib as initial therapy for advanced breast cancer. NPJ Breast Cancer 2019;5:5.

13. Johnston SRD, Harbeck N, Hegg R, et al. Abemaciclib Combined With Endocrine Therapy for the Adjuvant Treatment of HR+, HER2-, Node-Positive, HighRisk, Early Breast Cancer (monarchE). J Clin Oncol 2020;38:3987-98.

14. Tolaney SM, Wardley AM, Zambelli S, et al. Abemaciclib plus trastuzumab with or without fulvestrant versus trastuzumab plus standard-of-care chemotherapy in women with hormone receptor-positive, HER2-positive advanced breast cancer (monarcHER): a randomised, open-label, phase 2 trial. Lancet Oncol 2020;21:763-75. 